\title{
Automated Acquisition and Analysis of Selected Area Electron Channeling Patterns in an FEG-SEM
}

\author{
Joseph Tessmer ${ }^{1}$, Saransh Singh ${ }^{1}$, Yoosuf N. Picard ${ }^{1}$, Marc DeGraef ${ }^{1}$ \\ ${ }^{1}$ Dept. of Materials Science and Engineering, Carnegie Mellon University, Pittsburgh PA, USA
}

Fine details of crystalline structure, including local lattice parameters, shifts due to strain, and compositional variation, can be investigated by analyzing the positions of higher-order Laue zone (HOLZ) lines. Micrographs displaying these lines are often captured by convergent-beam electron diffraction (CBED), an imaging modality in the TEM. However, these lines can also be acquired in selected-area electron channeling patterns (SACP), a diffraction modality in the SEM that makes use of backscattered electrons. SEM holds several advantages over TEM, including simpler operation and minimal sample preparation, as well as the ability to analyze larger sample areas.

SACPs differ from conventional electron channeling patterns in that the point of incidence of the beam on the sample surface does not change over the course of the beam rocking. Rather, the angle of incidence is varied while the beam pivots on a single spot. Because of the potentially small size of this selected area, local changes to the lattice parameters can be identified by observing changes in the positions of HOLZ lines. Prior work has established a dictionary-based mapping technique for SACPs, which is used to identify an orientation for any given SACP based on the imaging parameters as well as the sample structure and composition [1].

Dictionary indexing of SACPs is accomplished by simulating patterns for all possible Euler angle combinations, with some finite step size in orientation space, and taking the dot product of experimental and simulated patterns [2]. The maximum value of this dot product is then taken to be the best fit for the experimental pattern.

Automated acquisition of SACPs over a large area is of interest for use in the characterization of materials with compositional gradients, fine grain structures, or variations in defect morphology. Using scripts to interface with the microscope, automated acquisition of patterns over a large grid has been demonstrated. The step size on such grids is limited mainly by the size of the selected area and the precision of the microscope stage. As such, tuning of the microscope to reduce the size of the selected area is an ongoing process, in addition to assessing the accuracy and repeatability of stage movement [3].

Once channeling patterns for each point have been captured, dictionary mapping provides Euler angles for each point, which can then be used to produce an inverse pole figure (IPF) orientation mapping similar to that obtained from electron backscatter diffraction (EBSD). Demonstrating automated acquisition and indexing of SACPs is a necessary step towards automated imaging of crystalline defects. Such imagining requires analyzing SACPs and tilting to a specific $\mathbf{g}$ vector to highlight dislocations. Future work will implement automated tilting and rotation for such dislocation imaging. Comparison of experimentally produced and indexed SACP orientation maps with EBSD IPF maps show similar results for both techniques [4]. 


\section{References:}

[1] Singh, S., De Graef, M., Microscopy and Microanalysis (2017, in press)

[2] Callahan, P. \& De Graef, M., Microscopy and Microanalysis 19 (2013) p.1255.

[3] Picard, Y. et al, Microscopy Today 20.02 (2012): p.12.

[4] Research supported by an ONR grant, \# N00014-16-1-2821. The authors acknowledge use of the Materials Characterization Facility at Carnegie Mellon University supported by grant MCF-677785.
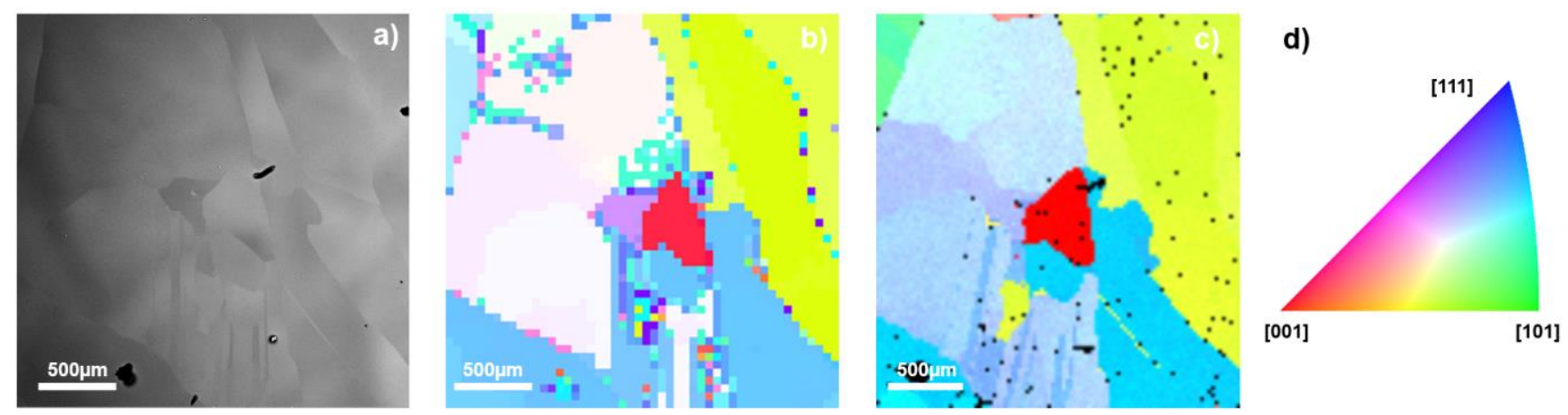

Figure 1. a) Secondary electron, b) SACP IPF map, and c) EBSD IPF map of the same area of a multicrystalline silicon sample. d) shows the IPF color map
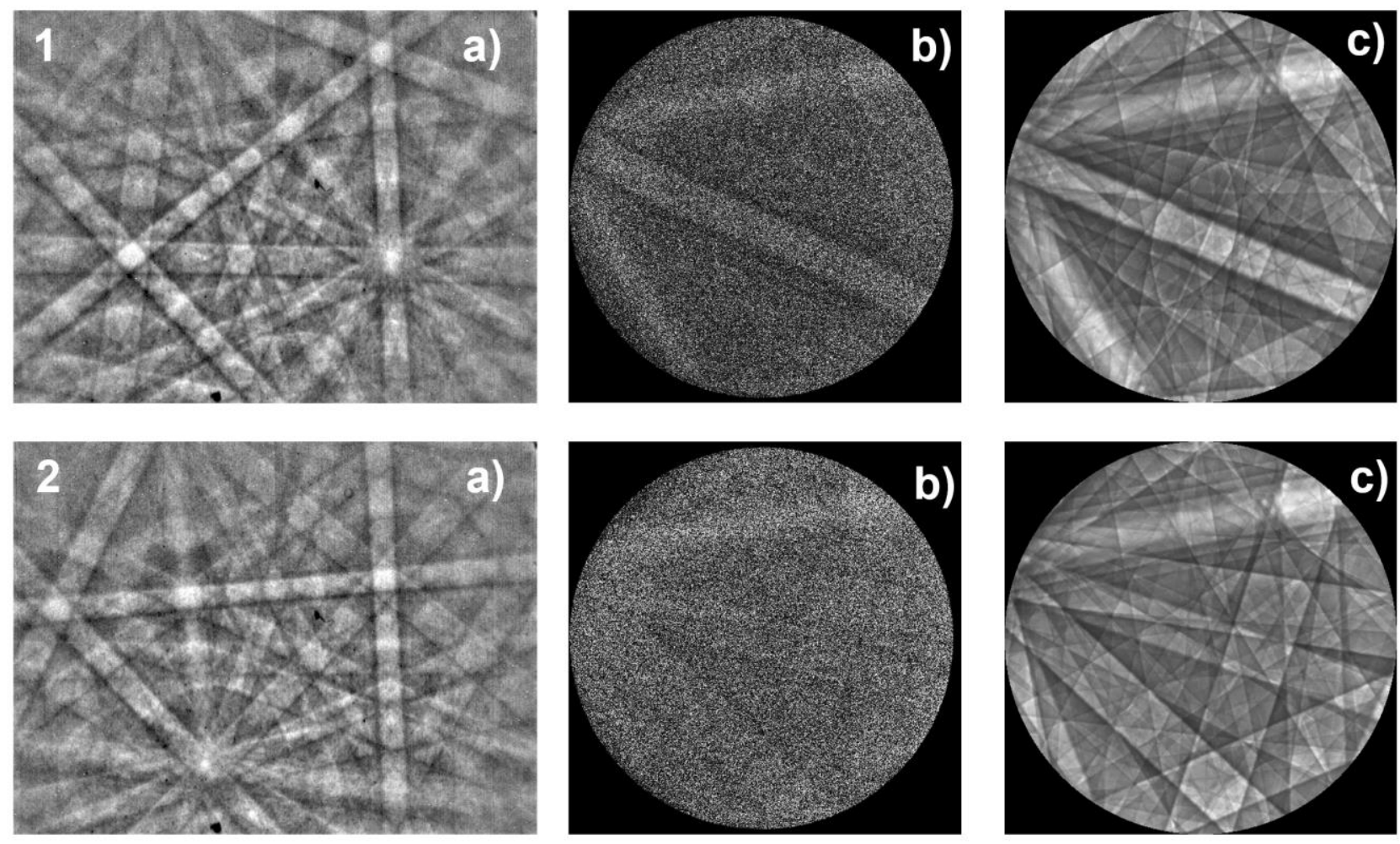

Figure 2. a) Experimental EBSD patterns from the SEM, b) Experimental SACPs from the SEM, and c) simulated SACPs indexed using the dictionary method. SACPs have a max. angle of $10.3^{\circ} .1$ and 2 denote adjacent grains on a multi-crystalline silicon sample. 